\title{
Vital Signs Measurement
}

National Cancer Institute

\section{Source}

National Cancer Institute. Vital Signs Measurement. NCI Thesaurus. Code C49672.

The act of assessing an individual's current temperature, heart rate, respiratory rate, pulse oxygenation, and blood pressure. 